\title{
Is astronomy useful for societies?
}

\author{
Jean Audouze \\ Institut d'Astrophysique de Paris \\ 98 bis, Boulevard Arago, 75014 Paris, France \\ email: audouze@iap.fr
}

\begin{abstract}
We briefly review various aspects related to the usefulness of astronomy to societies. Astronomy constitutes an important part of human culture, all over different countries and all over the entire history of humankind. Because of its intrinsic interest, astronomy is able to attract young generations towards technical and scientific careers. Moreover, it is also an indirect trigger of innovation and consequently of financial profit.
\end{abstract}

Keywords. Society, technology, telescopes, ITER

\section{Astronomy is the most ancient scientific discipline}

The oldest written remains do show that our ancestors were observing the skies and made use of these observations to build up calendars and to attempt to relate terrestrial events with the occurrence of celestial phenomena like the appearance of comets or novæ (stars which appear suddenly in the sky). The first astronomers were also supposed to make predictions. This explains the origin of astrology which still induces interest among many people although it has been proved that their so-called "predictions" have been found to be meaningless. The days were defined by the succession of light and night; the months correspond almost to the cycles of the Moon and the year is the whole cycle of the relative movements of the Earth and the Sun. The first calendars were, indeed, based on such observations.

Most religions have produced stories telling the origin of the world: this is the case, for instance, of the genesis which begins the Bible or of the Metamorphoses by Ovid. Visible stars seem to form some sorts of drawings in the night sky which are the constellations. Most of the names of the constellations visible in the northern hemisphere come from the Greek and the Roman mythology.

\section{The history of astronomy is closely related to that of humanity}

During the antiquity and the Middle Age, the universe was believed to be geocentric $\dagger$. Although the Ptolemaic theory attempting to describe the apparent movement of the planets in the sky was found to be wrong after the Copernican revolution, its life time has been more than 15 centuries long. The first astronomical revolution occurred indeed with the publication of the book De revolutionibus orbium caelestium libri sex by Copernicus, a few days before his death on 1543 where he transformed the geocentric vision into an heliocentric one. This first revolution in astronomy is pursued during the 17th century with the use of the first astronomical lens by Galileo on $1609 \ddagger$. Kepler publishes his laws concerning the rotation of the planets around the Sun on 1609 and 1619. From these

$\dagger$ With the exception of Aristarcos of Samos who was the first to claim for a time that the planets were rotating around the Sun.

$\ddagger$ The International Year of Astronomy took place in 2009 to celebrate the 400th anniversary of this event. 
relations, Newton deduces his theory concerning the gravitation which is published on 1687. Meanwhile, as a quite unknown French priest named Nicolas Cassegrain, Newton invents the principle of the telescope. The second astronomical revolution occurred during the first part of the 19th century with the invention of the photography by Niepce on 1827 and Daguerre on 1835. Bessel was one of the first to apply this technique to astronomy. This allows indeed to keep tracks of the observations by storing the images obtained by this way. At the same period, the German physicists Fraunhofer, Kirchhoff and Bunsen demonstrated that the lights emitted by the Sun and the stars, when they are dispersed by prisms or gratings, contain a lot of different lines, all of them characterizing a given chemical species either as an ion or as an atom. By analyzing such optical radiations one can deduce the chemical composition of the emitting or absorbing material. The spectroscopy and spectrography were thus born such as astrophysics which is the part of astronomy involved in the physical and chemical studies of the celestial bodies.

The third revolution in the history of astronomy took place at the beginning of the 20th century with the advent of quantum mechanics on 1900 by Planck and the related fields which are atomic and nuclear physics. At the same period, Einstein established the basis of special (1905) and General (1917) relativity which superseded the Newtonian theories of Gravity. Near Los Angeles on Mount Wilson, a large (2 m. diameter) telescope was built at the same time which allowed Shapley to demonstrate that the centre of the Milky Way is far away from the Sun at a distance of 30000 light year in the direction of Sagittarius, marking then the end of heliocentrism. With the same instrument, Hubble established on 1929 his famous relation between the recession velocity of external galaxies and their distance, showing that the observable universe is expanding.

The fourth revolution occurred on 1957 with the Soviet launch of the Sputnik satellite. It is the beginning of the spatial era : year after year, astronomers are able to build up detectors which are sensitive to electromagnetic radiations which are more energetic (Gamma, X and UV radiations) or less energetic (infra red, millimetric) than the visible and which are absorbed by the terrestrial atmosphere. Many space missions embarked these detectors and led to unprecedented views of the sky coming from these different parts of the electromagnetic radiation spectrum. With other satellites, the American cosmonauts walked at the surface of the Moon; the whole Solar System is deeply explored. Concerning the visible radiations, the American (NASA) and European (ESA) consortium was able to launch the Hubble Space Telescope (HST) on 1990 which provided the world with an unbelievable collection of astronomical data. At the same time the astronomers were able to use a new class of ground-based optical telescopes of the 8 to $10 \mathrm{~m}$ - diameter ranget. Let us quote in particular the two Keck telescopes located on the summit of Mauna Kea (on the Big Island of Hawaii), belonging to the University of California and the California Institute of Technology (Caltech) and the four $8 \mathrm{~m}$. telescopes being the Very Large Telescope (VLT) of the European Southern Observatory (ESO) and situated on Mount Paranal at the North of Chile.

We are presently awaiting the fifth revolution when astronomers will be able to detect easily external planets comparable to Earth, when they will detect and analyze the gravitational waves produced during the most violent astrophysical phenomena (explosion of supernovae and hypernovae; matter accretion on big black holes; ... ), when they will have access to the telescopes of the $30-100 \mathrm{~m}$. diameter range and when they will establish some astronomical observatories at the surface of the Moon which has no atmosphere.

$\dagger$ Remember that the sensitivity of a telescope is proportional to its diameter. In the interval 1947-1977, the biggest telescope was the $5 \mathrm{~m}$ - diameter one on Mount Palomar at the south of California ; from 1977 to 1990 it was the 6 m- telescope of Zelentchuk in the Soviet Union. 
From this very rapid and incomplete evocation one notices that the progresses in astronomy, especially during the recent past, now and for the future depend very much on those achieved by the technique, the engineering and the computing sciences. To exemplify this affirmation, let us quote the tremendous improvements in the observation capabilities obtained thanks to the so called adaptative optics where the aberrations and other optical defaults of the telescopes and their detectors are corrected instantaneously by specific computing programs. The infrared astronomy relies on the cryogenic techniques which make the noise in the detectors as low as possible. Thanks to all these technical improvements, we are living now in what we can call the golden age of astronomy.

\section{Some of the philosophical consequences of astronomy}

Astrophysicists and specifically those who study the Universe as a whole and who are named as cosmologists, provide interesting answers to some of the questions which belong to philosophy and metaphysics. The question of the origin is the topic of many cosmological programs. The current views deduced from the well accepted Big Bang theory are such that the age of the Universe is given as 13.7 billion years. This is the time elapsed since the original event when the Universe appeared very hot and dense and blew up since then.

The comparison of (i) the spectrum of the irregularities of the $2.7 \mathrm{~K}$ black body relic radiation observed by the space mission WMAP (for Wilkinson Microwave Anisotropy Probe) launched by NASA on 2001†, (ii) the expansion of the Universe measured by the extragalactic SN1a distribution observed by HST and (iii) the outcome of the Big Bang nucleosynthesis responsible for the formation of $\mathrm{D},{ }^{3} \mathrm{He}$ and ${ }^{4} \mathrm{He}$ and ${ }^{7} \mathrm{Li}$, allows one to determine the very nature of the energy-matter content of the observable Universe. According to this comparison, $70 \%$ of this content is a primordial dark energy, $27 \%$ is made of invisible (dark) matterł. The ordinary (nuclear) matter which constitutes the stars, the Sun and the whole Solar System represents only 3\% of the total content of the Universe $\uparrow$.

Astrophysics provides the techniques allowing us to determine not only the age of the Universe but also that of any star and specifically that of the Sun and the Solar System, equal to 4.555 billion years. It is also known that the overall life time of our Solar System should be of the order of 10 billion years: the Sun will become a Red Giant swallowing the Earth in a time comprised between 4 and 5 billion years. We know also that the whole Solar System including the Earth and ourselves is made of stellar ashes: Most of the chemical species constituting our bodies and our surroundings have been manufactured inside those stars having completed their evolution before the formation of the Solar System\|. My colleague Michel Cassé likes to say that a glass of water summarizes the origin of the ordinary (nuclear) matter: the atoms of Hydrogen are primordial while those of oxygen have been form not too long before the formation of the Solar System. Briefly speaking, we are the ashes of massive stars evolving very rapidly and using the energy coming from a low mass star (the Sun) evolving very slowly.

$\dagger$ ESA launched on 14 May 2009 the space mission Planck surveyor which aims to analyze these anisotropies with an accuracy 10 times better than that of WMAP.

$\ddagger$ This dark (invisible) matter is made of particles which have no electromagnetic interactions with our ordinary (nuclear) matter but which proves its presence by its gravitational effects.

9 The interested reader may consult the proceedings of the meeting entitled The Invisible Universe, organised in July 2009 at the UNESCO headquarters by Jean-Michel Alimi.

$\|$ With the notable exception of $\mathrm{H}$ and $\mathrm{He}$ which are of primordial origin. 
Another important philosophical question which can be expressed as Are we alone? can be considered by the astronomers. The search for an answer to this specific question involves three different approaches:

- the search of planets out of the Solar System: Since the first discovery of a jovian planet rotating around 51 Pegasi by Michel Mayor and Didier Queloz from the Geneva Observatory, more than 500 different external planets have been detected since then, most of them being jovian as the first one but with the expectation to detect many terrestrial ones in a very near future thanks to the COROT space mission launched on 2006 by CNES and forthcoming other missions like DARWIN of ESA which is scheduled to be launched around 2015 .

- the organic and biological chemistry analyses of cometary, martian samples and later coming from the ocean located inside the icy crust of Europa. Let us quote the space mission ROSETTA launched on 2004 by ESA which is expected to land on the nucleus of the comet $67 / \mathrm{P}$ Churyumov-Gerasimenko in 2014 and during two more years analysing the chemical composition of the matter of this nucleus. Several space missions called Mars Sample Return are under study by NASA and ESA. They aim to land on Mars and to return on Earth after having captured some samples taken from the ground of Mars. The presence of water at the surface of Mars is a positive sign in favour of a possible discovery of living tracks in these samples. This hopefully should occur during the 2010 years. About 20 years later, the space community contemplates the possibility to launch a satellite on Europe with the aim to return to Earth with samples coming from the inside ocean of this Galilean satellite of Jupiter. Should one of these searches resulting in a positive response, this would be a strong argument in favour of an external origin of life†.

- A few teams of radio astronomers like the one led by Jill Tarter from Berkeley are continuously scanning the sky in the hope to detect an intelligent signal. This type of program is called SETI (for search of an extraterrestrial intelligence). Given the fact that the distance between the closest possible intelligence and us should be big (more than a few 1000 light years), the success of this type of program can only rely on luck.

\section{We do expect considerable progress in technology coming from astronomy}

I have written above that most of the recent progresses in astronomy comes from the overwhelming developments in technology. It seems fair to claim that many of these developments have been triggered by the demands of the astronomers. Let me give a few examples:

- The observing facilities becoming bigger and bigger challenge the companies hired to build them and force them to innovate in these building processes: for instance, the stability of such facilities has to be achieved at a very high level of performance.

- Astronomy is not only done with telescopes looking at the sky but also with devices buried inside very deep mines or in the depth of oceans. This is the case of detectors aiming to collect neutrinos or cosmic rays. The high performance needed for such detectors is another constraint forcing the industrial companies involved to be able to reach such a high level of performance.

$\dagger$ The origin of life is said to be internal if the first primitive living beings result in processes which have occurred on earth during its first evolution phases ; it is said to be external if these living beings come from seeds coming eg from comets or from meteorites. 
- As said before, one needs to call on the highest quality cryogenic devices. In some cases one has to make use of very large magnetic fields. These requirements are another incentive for the companies working in such domains.

- The computing capacities have to be very large (astronomy deals presently with the teraflop domain). Computers are used in many subfields by astronomers: (i) to orient the telescopes and the satellites; (ii) to make use of optical adaptations; (iii) to perform remote observations: an astronomer living in Paris being able to direct from his office a telescope located in Hawaii; (iv) to reduce and interpret the huge flow of observational data coming from the ground or from space.

- It is interesting to note that astronomers have made use of CCD detectors at the focus of their telescopes a long time before that the commercial TV employs this type of detectors in their cameras or their receiverst.

- In a somewhat related field, some of my colleagues working at the Meudon Observatory collaborate with Prof. José Sahel, a M.D. of the Quinze Vingts Hospital in Paris to build up some artificial retinas allowing some blind people to recover in part their vision.

- It is also well known that some of the detectors used in radio astronomy are similar to the scanners used in medicine.

- Astronomy also induces the outcome of terrestrial or human devices. Some are built for military reasons like the nuclear weapons which mimick what occurs in exploding stars like supernovae; France and Us are currently building facilities inside which a mixture of $\mathrm{D}$ and $\mathrm{T}$ will ignite after being bombarded by a large set of very powerful lasers. The French facility is located near Bordeaux and the American one is inside the Livermore laboratories. Some other applications are civilian: this is the case of the ITER (International Thermonuclear Energy Reactor) international project located in Cadarache (south of France). A mixture of D and T compressed and heated by very large and efficient magnets should be able to fuse in ${ }^{4} \mathrm{He}+n$. The purpose of ITER is to reproduce a nuclear reaction which takes place inside the Sun and which provides some of its energy. Should ITER be successful, it will be another step towards the use of the fusion nuclear reactions as an energy provider.

To sum up, many technological innovations are boosted by astronomy with the related economical benefits.

\section{How to make astronomy a popular field?}

The main goal of the celebration of the International Year of Astronomy (IYA09) which prompted the organisation of such a IAU symposium is an attempt to convince everybody whatever his (her) age, his (her) culture, his (her) country, that it is important for him (her) to look at the sky, to admire its beauty and to be knowledgeable in a very simple basis of the main astronomical notions (stars and planets, Milky Way and other galaxies, ...). At the time of the writing of this text, I do believe that this goal has been widely and successfully achieved.

One should encourage all the nations to organize the teaching of the simple notions of astronomy in the curriculum of elementary and high schools and of the colleges of all students learning in such establishments. There are indeed many journals and reviews, many popular books and textbooks dealing with astronomy and its discoveries. There might not be enough radio and TV programs such as not enough movies concerning the observation of the skies. Radio, TV and movie producers should be encouraged to

$\dagger$ CCD means charged coupled devices. These detectors are made of a matrix of $n$ times $m$ pixels. The astronomers make a large use of them since the 1970s. 
look in that direction. Hopefully, the interested individuals have access to many internet sites devoted to astronomy. The main observatories and space agencies make a lot of exciting documents at the disposal of those who use this channel of information. Most of the scientific museums possess their planetarium which is a very attractive element of their program. From time to time, some exhibitions are realized on those topics. Let me quote, for instance, the most successful permanent exhibition entitled The Great History of the Universe produced by the Cité des Sciences et de l'Industrie (La Villette) in Paris. There are also several astronomical festivals like the one organised in August every year at Fleurance, a small city in the South-West of France. Every year, in France, each city and/or club of amateur is encouraged to organise its Nuit des Etoiles around the middle of August when there are many shooting stars in the sky. This year, the organizing committee of IYA09 has proposed One hundred hours of observations in April. The Nuits des Etoiles takes place during the end of July to benefit from the absence of the Moon in the night sky. Finally, there are sessions of observations during October when the Galilean satellites of Jupiter - which have been first observed by Galileo with his astronomical lens- are be conspicuous in the 2009 sky. The existence of many clubs and associations gathering the amateurs in astronomy plays a major role in the popularisation of this scientific discipline.

\section{A few final remarks}

The observation of the skies is one of the basic curiosities shared by everybody. In that respect I am always astonished by the overwhelming interest shown by all the publics when a solar eclipse occurs; a scientific experiment based on the Newtonian theory of gravitation can be appreciated by everyone. We, professionals have the duty to recall as often as possible that the sky belongs to everybody.

I hope that we are all convinced that astronomy constitutes an important part of the human culture, all over the different countries and all over the entire history of mankind. Because of its intrinsic interest, Astronomy is able to attract young generations towards the technical and scientific careers. I can testify it by many examples which occurred during my career. Moreover, it is also an indirect trigger of innovation and consequently of financial profit. 